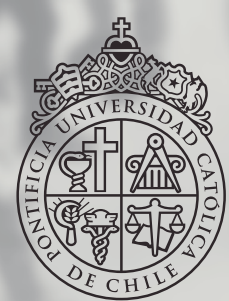

PONTIFICIA UNIVERSIDAD CATÓLICA DE CHILE

\title{
Revista
}

Desigualdades de género en el emprendimiento y en los negocios de las mujeres.

Carola Naranjo

Familias y servicios, coordinados para encontrar soluciones eficaces a los problemas de protección de la infancia.

El modelo de las

Family group conferences.

FRANCESCA MaCI
Acción materna y acción social: el caso estadounidense. MARÍA José BinetTI

\section{La búsqueda del} paraíso:

el apego y la reparación de la infancia perdida en niños vulnerados en sus derechos

CAtherine Anne LaBrenz

\section{Posibilidades y} límites del enfoque de intervenciones sociales basadas en evidencia

ENTREVISTA

Dr. CHRISTOPHeR BoNell 



\title{
Familias y servicios, coordinados para encontrar soluciones eficaces a los problemas de protección de la infancia. El modelo de las Family group conferences
}

Family services: liaising to find effective solutions for child protection problems. The family group conference model

\author{
Francesca Maci \\ Investigadora y doctora en ciencias de la Organización y Gestión de la Universidad Católica de Milán. Se desempeña además como \\ trabajadora social en el trabajo directo con niños y familias colaborando en también en la la formación y supervisión de servicios \\ sociales locales y del tercer sector en materias de protección de niños niñas y adolescentes. francesca.maci@unicatt.it
}

\begin{abstract}
Resumen
Un trabajo social que pretenda la real protección de la infancia está, antes que todo, orientado a proyectar amplias redes de ayuda, capaces de generar bienestar para los niños y jóvenes que se encuentran viviendo situaciones dificiles en sus familias. Un modelo innovativo que puede venir en ayuda en ese sentido es aquél de las Family Group Conferences (FGC) que propone un proceso relacional, acompañado por la figura llamada facilitador, en el que la familia extensa y otras personas significativas de los servicios públicos para las familias, toman decisiones en favor de los niños y jóvenes que requieren la preparación de un proyecto de protección y cuidado para garantizar su bienestar. Tal acercamiento quiere subrayar cómo las intervenciones en las que son exclusivamente los profesionales los que deciden, llevan a la familia a asumir una actitud pasiva de delegación con respecto al enfrentamiento de la situación. La Family Group Conference constituye un válido antídoto para este riesgo porque mira a la familia como la protagonista principal de su recorrido de cambio, considerándola potencialmente dotada de fuerzas y capacidad para generar soluciones positivas para la vida de niños y jóvenes. Se exponen las fases del modelo desarrollando el proceso de un caso.
\end{abstract}

Palabras clave. Protección, infancia en dificultades, modelo, familia, redes, facilitación, participación.

\begin{abstract}
A social work seeking the real protection of children is, first of all, aimed at projecting broad support networks, capable of generating welfare for children and youth who are living in difficult family situations. An innovative model that can come in help in this regard is that of the Family group conferences (FGC) proposed a relational process, accompanied by the figure called facilitator in which the extended family and significant others of public services for families make decisions on behalf of children and young people who require the preparation of a project to protect and care to ensure their welfare. Such an approach would emphasize how interventions that are exclusively professionals who decide, take the family to take a passive attitude delegation on coping with the situation. The Family Group Conference is a valid antidote to this risk because look at the family as the main protagonist of his journey of change, considering potentially endowed with strength and ability to generate positive solutions to the lives of children and youth. Stages of developing the exposed pattern of an event process.
\end{abstract}

Key words. Protection, Children in difficulty, model, family, networks, facilitation, participation. 


\section{Premisa: protección de la infancia y participación}

Todo profesional comprometido en el ámbito de los servicios de protección de la infancia, interesado por desarrollar de la mejor manera su trabajo, está llamado a preguntarse:¿qué significa trabajar para la protección de niños y jóvenes?, y a tratar de encontrar respuestas que vayan más allá de la retórica superficial.

Un trabajo social que pretenda la real protección de la infancia está, antes que todo, orientado a proyectar amplias redes de ayuda, capaces de generar bienestar no solo para los niños y jóvenes, sino también para sus padres en la dirección de un cambio familiar integral. Coherente con el deseo de hijos, padres y madres de volver a vivir una buena vida juntos.

Para ser verdaderamente protectoras, las intervenciones puestas en el campo por los profesionales deberían encontrar una significativa correspondencia en el mundo de la vida de adultos, niños y jóvenes en dificultades. La sustentabilidad de los procesos de ayuda en el ámbito de la protección de la infancia requiere, en efecto, de la implicación de los directos interesados que se sentirán mayormente motivados a recorrer un camino si han contribuido efectivamente a escogerlo, si se perciben con capacidad de recorrerlo con confianza más bien que obligados a emprenderlo (Folgheraiter, 2006 y 2007). Un proyecto de ayuda, aún cuanto sea irreprochable en el plano técnico-profesional, sirve de poco si no es comprendido por quien debe ponerlo en práctica y si resulta difícilmente "manejable" en el contexto concreto de la vida cotidiana de las personas involucradas en el problema (Ferguson, 2001; Maci, 2008; Parton, O’Byrne, 2000).

Para una "buena protección", coherente con la dimensión plural propia del trabajo social, otro aspecto imprescindible es el involucramiento de las personas significativas que gravitan en torno a la familia, a menudo ya comprometidas en el intento de hacer frente a las dificultades del vivir. Es indispensable ensanchar la mirada y poner a la familia y su red de relaciones en el centro de los procesos decisionales que tienen como objeto la protección de la infancia, con el fin de encontrar estrategias de intervención mayormente eficaces, pero sobre todo menos opresivas (Dominelli, 1998).

En el marco apenas delineado, el trabajo social desplegado por los profesionales en el ámbito de la protección de la infancia no puede más que estar orientado a facilitar el enfrentamiento de los problemas existenciales por parte de las personas, de las familias $y$ de la comunidad involucradas, encauzando su fuerza reflexiva y sus energias motivacionales directamente sobre aquellas contigencias incómodas [...] con el explícito objetivo de mutar las preeocupaciones de las personas con respecto a sus existencias en energía humana transformadora de las mismas (Folgheraiter, 2011).

Pero para evitar quedarse en el plano de la pura retórica y contentarse con utilizar el concepto de participación de las familias como un mero eslogan, es necesario individualizar recorridos e instrumentos operativos que permitan redireccionar las lógicas de poder en sentido democrático, reemplazando la supremacía de la ténica con la paridad de los saberes. Un modelo innovador que puede venir en ayuda en ese sentido es el de las Family Group Conferences (FGC) (Marsh e Crow, 1998; Ashley e Nixon, 2007). Son un acercamiento de tipo participativo (Connolly, 1999; Merkel-Holguin, 2004), de amplia difusión en el panorama internacional, que permite trabajar en conjunto a las familias en un ámbito tan complejo y desafiante como el de la protección de la infancia.

\section{El modelo de las Family Group Conferences}

El modelo pone a la familia ${ }^{1}$ en el centro del proceso decisional que le atañe (Burford, 2010), valorizando, también en una situación de dificultad, la expresión de su agency (Archer, 2003) en definir, en colaboración con los operadores de los servicios de protección de la infancia, estrategias de intervención sensatas y útiles para enfrentar las dificultades presentes.

Las Family Group Conferences son sintéticamente definibles como un proceso de decision making (Morris, Burford, 2009) orientado a valorizar la capacidad de la familia para enfrentar los problemas que encuentra en el curso de la propia vida, en particular en el cuidado y en la atención de los niños, a través de tomar decisiones autónomas acerca de las posibles soluciones que hay que emprender para abordar la situación de dificultad que enfrenta (Burford, 2001; Maci, 2011).

Concretamente, son un encuentro entre los componentes de la familia, los operadores de la protección de la infancia y otras personas cercanas al núcleo

1 La familia está entendida en sentido amplio; además de la red parental incluye las relaciones significativas cercanas al menor y a sus padres, en grado de sostenerlos en el enfrentamiento de las dificultades presentes. 
familiar, dirigido a proyectar intervenciones de protección y cuidado de niños y jóvenes considerados en situación de riesgo o perjuicio. En el curso de dicho encuentro nos confrontamos con las preocupaciones presentes y con las estrategias que se pueden poner en acción para hacer frente a las dificultades de los jóvenes y mejorar la situación familiar. En la FGC participan los miembros de la familia ampliada definida según una concepción extensa, que vuelve a incluir los lazos significativos -parentales o no- disponibles para sostener al menor y sus padres a través de una ayuda concreta.

\section{La historia y los ámbitos de desarrollo}

El modelo nace en Nueva Zelanda a fines de los años ochenta en el ámbito de la child protection, como resultado de un proceso dirigido a contrabalancear el poder de los profesionales en la gestión de las situaciones de protección de infancia con la valorización de la cultura tribal maorí y la capacidad propia de la familia ampliada de ocuparse de sus miembros más frágiles. Los principios de referencia se han desarrollado a partir de la crítica según la cual en las evaluaciones y en los procesos decisionales se atribuía mayor peso a la opinión de los profesionales, de los abogados y de los administradores de los servicios, debilitando las capacidades de la familias y de las comunidades maoríes. Las Family Group Conferences nacen, por lo tanto en el contexto neozelandés como un proceso cultural dirigido a valorizar las capacidades de la familia, aún cuando se encuentre viviendo momentos de fuerte dificultad que ponen en crisis sus competencias en el cuidado de sus miembros más pequeños. Esta perspectiva ha sido difundida desde que Nueva Zelanda aprobó su propia ley sobre la protección de la infancia, en noviembre de 1989.

Según un estudio del año 2005 (Nixon, Burford, Quinn, Edelbaum) las Family Group Conferences se utilizan en diecisiete países. Este acercamiento es utilizado en muchos países del norte de Europa, el Reino Unido, la República de Irlanda, el este de Europa, Australia, Canadá, los Estados Unidos y Sudáfrica.

La utilización de las Family Group Conferences resulta apropiada en ámbitos y contextos en los cuales es necesario asumir decisiones y definir proyectos de intervención (Morris, 2004). El modelo, basándose en un acercamiento de tipo participativo y de valorización de las competencias y del saber derivado de las experiencias de las familias, es fácilmente adaptable a las diversas situaciones problemáticas que puede encontrar una familia. Por esta razón, a pesar de nacer en el ámbito de la protección de niños y jóvenes, ha encontrado difusión en otros ámbitos del trabajo social entre los cuales está el penal juvenil, la escuela, la violencia doméstica, las separaciones, los adultos vulnerables, la salud mental y los ancianos.

\section{Los actores involucrados en la Family Group Conference}

Además de la familia, protagonista indiscutida del proceso, son diversos los actores que toman parte en la Family Group Conference y que colaboran activamente en su realización. Entre estos un rol crucial está cubierto por el facilitador (Ashley, Holton, Horan, Wiffin, 2006; Connolly, 2006) y por el advocate (Dalrymple, 2002).

El facilitador es el sujeto al cual se confía la dirección del proceso. Se ocupa de la gestión de la Family Group Conference desde el inicio hasta el final; desde la fase de preparación a la de facilitación propiamente tal del encuentro, con el objeto de sostener a la familia en su importante tarea de proyección. En sentido general, sus acciones están orientadas a involucrar al menor, a la familia, a los operadores de los servicios y a la comunidad, representada por las otras relaciones significativas que podrían contribuir a la elaboración del proyecto de protección.

Su característica principal es la de la independencia. En efecto, el facilitador no está involucrado en otros procedimientos decisionales que tienen que ver con la familia en cuestión. Es importante que sea considerado neutral con respecto a los operadores del servicio de protección de infancia como asimismo respecto de los familiares. No toma parte por ninguno; su interés preeminente es el buen logro de la Family Group Conference. Al facilitador no le son requeridas particulares competencias profesionales, sino que considerables habilidades en las relaciones interpersonales y cualidades personales que lo lleven a ser abierto, acogedor y que confíe en las capacidades de la familia para hacer frente a sus propios problemas. Un buen facilitador debe además poseer significativas habilidades organizativas. Otra figura relevante es el advocate, que en el curso del encuenro desarrolla la función de apoyar y sostener al menor en calidad de vocero. La finalidad principal de su operación es la de dar voz a los pensamientos y a las opiniones de niños y jóvenes para que sean escuchados y encuentren un lugar concreto en la elaboración del proyecto de protección. Puede ser un profesional del servicio de Family Group Conference, un volutario o un familiar. 


\section{El modelo de la Family Group Conference}

La FGC se desarrolla según un proceso estructurado (Fig. 1), que en su definición busca garantizar el derecho de la familia a participar activamente en los procesos decisionales que tienen que ver con ella, para encontrar soluciones adecuadas a los problemas. Se subdivide en cinco fases bien distintas, pero estrechamente conexas la una con la otra y que, para el buen logro del proceso en su conjunto, requieren de una acción sinérgica de varios actores involucrados.

\section{a. Activación}

Esta primera fase del proceso es fundamental porque una Family Group Conference se puede realizar solo a partir de la confianza de los operadores en esta modalidad de trabajo, orientada a valorizar y al mismo tiempo responsabilizar a la familia.
El proceso se pone en marcha con el envío por parte del servicio territorial, de acuerdo con la familia, de la ficha de activación al servicio de FGC. La ficha de activación está acompañada por una nota que contiene las informaciones útiles para la $\mathrm{RDF}^{2}$, documento en el que se indican:

- las principales informaciones con respecto a la familia;

- las preocupaciones presentes con respecto al niño (del servicio social y de la familia);

- los recursos a disposición (familiares e institucionales);

- una batería de preguntas centradas en la protección del menor, al cual los familiares reunidos en el encuentro deberán responder a través de la redacción del proyecto. Estas preguntas formula-

FIGURA $N^{\circ} 1$

LAS FASES DEL PROCESO DE FAMILY GROUP CONFERENCE

(modificado por Ashley et alter, 2006, p. 9.)

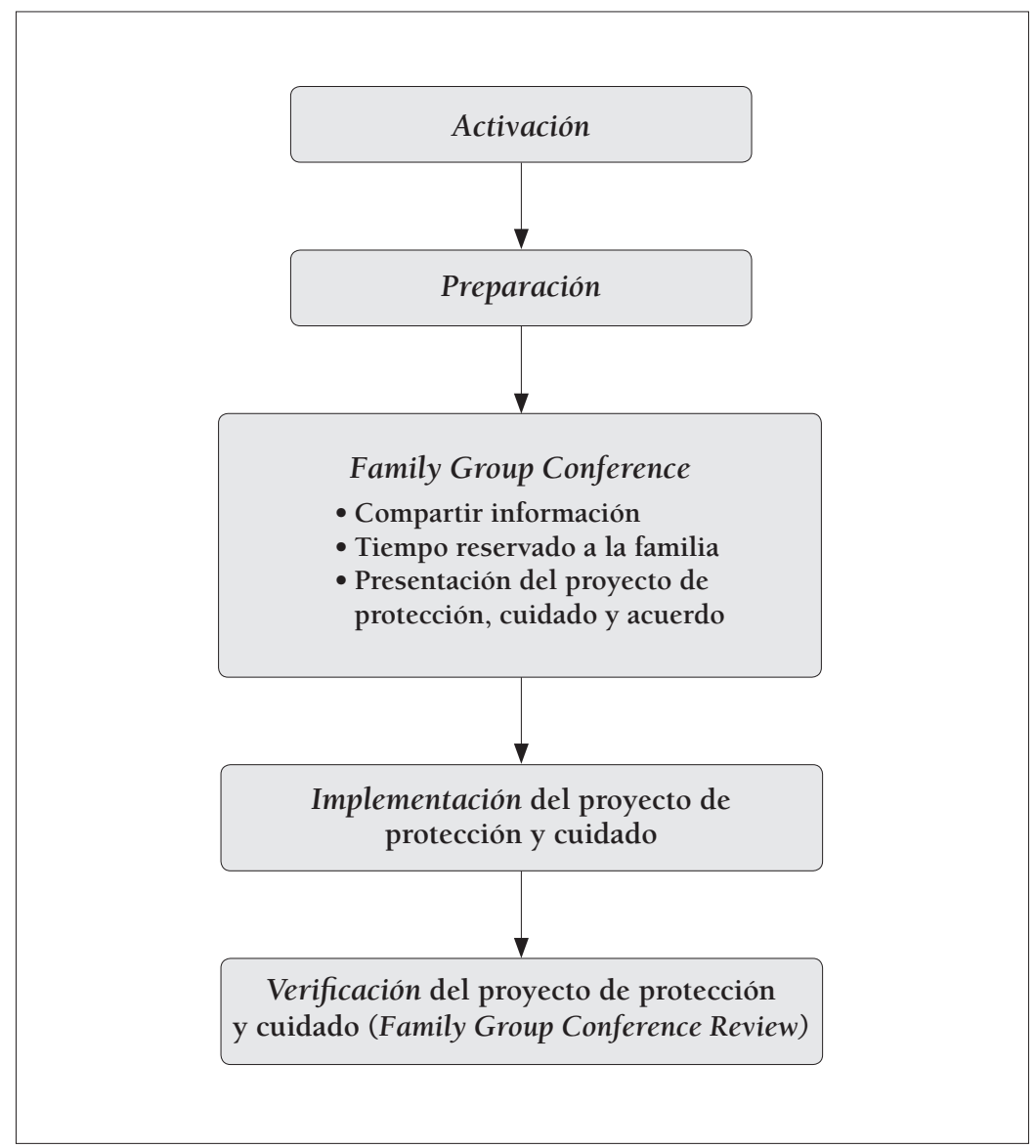

2 "Resource Description Framework (Descripción de la Estructura de Recursos). 
das por los servicios están pensadas para facilitar a la familia en el curso del proceso decisional.

\section{b. Preparación}

La fase de preparación de la Family Group Conference se considera crucial tanto para la familia como para los operadores que participarán en ella, porque caracteriza el recorrido completo de la Family Group Conference. El facilitador trabaja en estrecha colaboración con el menor y su familia para identificar la red familiar y las personas que desean invitar a la reunión. Se reune con los sujetos involucrados para explicarles en qué consiste la Family Group Conference, el sentido de su presencia y para recoger su punto de vista. Decide junto con los participantes las cuestiones organizativas vinculadas con la fecha, la hora y el lugar del encuentro.

\section{c. La Family Group Conference}

La Family Group Conference propiamente tal representa el corazón del proceso en su conjunto, porque es el momento en que todas las personas interesadas en la construcción de una situación de mayor bienestar para el niño se reúnen con el objetivo de elaborar un proyecto de protección eficaz y sostenible en favor de este y de sus padres. El proceso se subdivide en tres momentos principales:

1. Compartir información: en el momento de puesta en marcha del encuentro el facilitador presenta a todos los participantes, y cada uno de los presentes es llamado a explicar cuál es su relación con el menor de quien se deben ocupar. En la apertura del encuentro, los familiares y los profesionales presentes subrayan los elementos de riesgo y prejuicio que se atisban en la situación de vida del menor y de los padres, las informaciones que poseen acerca de la familia, sus tareas institucionales y los recursos a disposición en la redacción del proyecto. En esta fase se ponen las bases para la creación de un clima sereno y acogedor, basado en la colaboración y el apoyo recíproco, donde todos puedan percibir que cubren un rol relevante y paritario. En particular, la familia debe ver que los operadores están allí para ayudarla, más que para investigar o evaluar sus acciones y emitir un juicio a favor o en contra de su capacidad de cuidado;

2. Momento reservado a la familia: se deja sola a la familia para que pueda planificar con autonomía. Los participantes del encuentro deben individua- lizar un proyecto concreto en el cual concuerdan, el que deberá tener en consideración las cuestiones planteadas por el operador a la familia en la reunión de familia y las condiciones de cuidado consideradas imprescindibles (bottom line) para el servicio de protección de la infancia. La familia está llamada, además, a decidir cuándo y cómo revisar el proyecto elaborado después de un determinado período de tiempo. El facilitador, en esta fase, queda a disposición de la familia para apoyarla si se presentase necesidad de ello;

3. Presentación y acogida del proyecto: la familia expone, con la ayuda del facilitador, el proyecto a los profesionales presentes para su discusión y subscripción. El proyecto puede ser rechazado solo en el caso de que los servicios no lo consideren sufientemente protector para el menor. El facilitador se encargará de verificar que cada uno haya entendido los acuerdos tomados y entregará a la brevedad a todos una copia escrita del proyecto.

\section{d. Implementación y monitoreo}

Desde este momento en adelante se abre la fase de la implementación del proyecto. La protagonista es nuevamente la familia que debe activarse para realizar todo lo decidido, contando con el apoyo y la colaboración del servicio de protección institucionalmente competente, que aume un rol de guía del proceso y se hará cargo de monitorear que los compromisos tomados sean respetados efectivamente. El facilitador sale de la escena y se encontrará nuevamente con la familia para preparar la siguiente Family Group Conference de verificación.

\section{e. Verificación}

El proceso prevé una segunda FGC de verificación acordada en el curso de la primera reunión familiar. En este encuentro, similar al precedente en su funcionamiento, el proyecto es revisado, para verificar si ha funcionado, si son necesarias modificaciones o si es necesario intervenir de otro modo para alcanzar los objetivos prefijados.

\section{Estudio de caso: Lidia y Loris}

Las Family Group Conferences, además de ser utilizadas en diversos ámbitos de intervención social, pueden ser utilizadas en diferentes momentos del proceso de protección en curso para apoyar a la familia en la asunción de decisiones importantes para el propio recorrido de cambio.

La situación descrita ${ }^{3}$ nos será útil para comprender

3 La situación presentada se refiere a una familia que tomó parte en una Family Group Conference facilitasda por la autora, realizada dentro de un proyecto piloto implementado en Italia en el ámbito de la protecciónde de la infancia. 
mejor el funcionamiento del modelo en el campo y comprender su valencia positiva, aquello que el servicio social propuso a la familia que participó en la Family Group Conference para definir un proyecto de cuidado y protección para Lidia y Loris, esto antes de pedir el cierre del procedimiento en el Tribunal.

Lidia y Loris ${ }^{4}$ son dos hermanos de 15 y 11 años que, después de un período de alrededor de tres años transcurrido en una comunidad educativa para niños y jóvenes alejados de su casa, ahora viven nuevamente junto al padre Luis. El regreso a la familia ocurrió después de una evaluación positiva por parte de los servicios sociales de la trayectoria de cambio del padre, actualmente separado de la madre, acogida favorablemente por el juez referente de la situación.

En su momento, el alejamiento de los hijos del núcleo familiar había sido dispuesto por el Tribunal para protegerlos de la situación de riesgo presente en el contexto familiar, determinada por la relación fuertemente conflictual entre los padres y por dificultades con ellos.

La madre, desde la inserción en comunidad, no ha mantenido una relación constante con los hijos y actualmente sus contactos se limitan a llamadas telefónicas esporádicas.

Como apoyo a la progenitoriedad del padre, han sido activadas diversas intervenciones de apoyo, entre las cuales la inserción cotidiana en un centro educativo vespertino garantiza a los muchachos ayuda en el desarrollo de las tareas y un contexto relacional positivo. Loris está a cargo de una neuropsiquiatra infantil para enfrentar sus dificultades escolares y para un apoyo psicológico.

Luis y sus hijos tienen una relación significativa con su abuelo Walter, que es muy cariñoso con los nietos y representa un gran apoyo en su cuidado cotidiano. Lidia y Loris tienen una buena relación también con su tío Alfredo y su pareja Raffaella. Walter, en cambio, tiene un conflicto abierto con el hijo Alfredo porque no comparte sus elecciones de vida y, en particular, no aprueba la relación con su pareja Raffaella. Sus constantes peleas crean un clima tenso que pone en dificultades a los muchachos, que están muy ligados tanto al abuelo como al tío. Lidia y Loris, además, no tienen una buena relación con Stefania la conviviente del abuelo Walter porque es muy severa y los mira en menos.

Los servicios sociales creen que, en consideración al recorrido positivo de Luis y a sus buenas capacidades como padre, ha llegado el momento de proponer el cierre del procedimiento de protección en el Tribunal para restituirle plena potestad paterna. Permanecen, sin embargo, algunas preocupaciones por parte del servicio en relación con las tensiones presentes al interior de las relaciones familiares que corren el riesgo de limitar su capacidad de ocuparse de los hijos y de amenazar la serenidad del clima familiar.

En esta situación, la Family Group Conference aparece como una buena oportunidad para permitir a la familia hacer frente a las dificultades presentes ligadas a las relaciones conflictivas entre el abuelo y el hijo Alfredo, así como a aquellas de su conviviente Stefania con los muchachos. El encuentro entre los miembros de la familia podría ser útil para individualizar las estrategias que hay que poner en juego para contribuir recíprocamente a construir un clima sereno y de apoyo favorable al buen crecimiento de los muchachos.

El servicio social, además, a pesar de considerar fundamental el apoyo de Walter al hijo en el cuidado de Lidia y Loris, teme que por su tendencia a substituirle y al poner en la sombra su figura de padre, corra el riesgo de limitar de hecho su autonomía progenitorial. La asistente social finalmente estima que la Family Group Conference es una buena ocasión para comprender cómo la familia en su conjunto, colaborando recíprocamente, piensa sostener concretamente a Lidia, Loris y al padre en la gestión de lo cotidiano.

La asistente social está preocupada por la marcha escolar de Loris, como por la organización de sus jornadas y del tiempo libre de los muchachos y querría entender cómo desean reglamentar su relación con la madre que recientemente ha solicitado al servicio encontrarse con ellos.

La familia acoge favorablemente la propuesta del asistente social considerándola una oportunidad para que la madre pueda, después de unos años, volver a caminar autónomamente sobre sus propias piernas.

En la Family Group Conference tomarán parte:

- Lidia y Loris, que en el curso del encuentro estarán acompañados por una operadora de advocacy que los ayudará a dar expresión a sus pensamientos y sentimientos ante los adultos;

- el papá, Luis; 
- el abuelo Walter, primer invitado en la lista de los muchachos y de Luis porque es una presencia importante en su vida;

- el tío Alfredo y la "tía Raffaella" con quienes los muchachos están muy encariñados;

- Martina, la educadora del centro diurno invitada por Lidia y Loris como parte de la familia porque se ocupa de ellos cotidianamente;

- Mario, referente de la comunidad educativa invitado por el papá como miembro de la familia porque sin su apoyo y su ayuda se habría rendido y ahora no tendría a sus hijos en casa consigo;

- Beatriz, educadora de la comunidad invitada por los niños como miembro de la familia porque en los años que han estado lejos del papá ha sido una figura afectiva muy importante para ellos;

- Ana María, la asistente social del servicio de protección referente para la situación que ha propuesto a los muchachos y al padre que participaran en una FGC;

- Nora, la coordinadora del centro educativo invitada por la asistente social porque conoce bien a los muchachos, al padre y la situación.

El día de la Family Group Conference, el tío Alfredo y su pareja Raffaella han decidido no participar en el encuentro porque el día anterior tuvieron una encendida discusión con Walter.

La reunión de famila, que duró casi tres horas, concluyó positivamente porque los participantes lograron elaborar un Proyecto de Protección y Cuidado que garantizaba suficientemente protección, el que fue acogido favorablemente por la asistente social.

En particular el proyecto establece que:

- No obstante las dificultades presentes, Loris en septiembre permanecerá en la misma escuela pero cambiará de clase y en la primera fase de inserción será acompañado por un educador;

- Lidia y Loris seguirán frecuentando el centro educativo pero solo tres días a la semana porque desean pasar algunas tardes en casa. Será el abuelo el que los ayudareá a hacer las tareas y a verificar que todo vaya de la mejor maneras.

- Terminada la escuela seguirán yendo al centro educativo y participarán en las vacaciones organizadas por esta estructura;

- Loris no se siente capaz de hacer deporte. Lidia quiere ir a nadar a la piscina y el abuelo la acompañará cuando lo pida.
- El abuelo se compromete a hablar con su conviviente Stefania pidiéndole ser más amable con sus nietos y no entrometerse en su educación. Los muchachos se comprometen a ser mas educados con ella.

- Para Lidia, Loris, y el papá Luis, la ayuda del abuelo es indispensable y no la viven como una intrusión en su familia.

- Lidia y Loris no se sienten capaces de volver a ver a la mamá y piden que sea la asistente social quien le comunique la decisión de ellos; aceptando escucharla por teléfono, pero en presencia de los educadores del centro educativo.

La familia decide reencontrarse después de tres meses para verificar que lo que han decidido juntos se esté realizando de la mejor manera, y aportar los ajustes necesarios en caso de dificultad.

La Family Group Conference ha sido una ocasión para redescubrir, en un momento particularmente doloroso de la historia familiar, relaciones profesionales significativas, en las que han representado un sólido punto de referencia para los muchachos y su padre. Para ellos ver en la sala reunidas a algunas personas interesadas en su bienestar significa una experiencia de cercanía humana, sentimiento del que cada uno necesita para estar bien y construir una buena imagen de sí.

La reunión de familia representó la posibilidad de construir una visión común y de restituir al padre, a los muchachos y al abuelo una mirada de confianza por parte de la asistente social acerca de su futuro recorrido familiar.

\section{Conclusiones}

Como se ha evidenciado claramente en el caso descrito, las Family Group Conference se basan en que se debe asumir que la mayor parte de las familias, aun cuando están en dificultades, quieren hacer lo que sería bueno para sus propios miembros (Williams, 2004). Tal acercamiento quiere subrayar cómo las intervenciones en las que son exclusivamente los profesionales los que deciden, llevan a la familia a tomar una actitud pasiva de delegación y a no asumir una responsabilidad directa con respecto al enfrentamiento de la situación. De este modo, las intervenciones públicas, en vez de valorizar el potencial humano y relacional presente en lo social, arriesgan el bloqueo de los recursos presentes en las redes familiares, limitando de hecho su natural capacidad de ayudarse y de darse una mano recíprocamente. La Family Group Conference constituye un válido antídoto para este riesgo porque este 
acercamiento mira a la familia como la protagonista principal de su recorrido de cambio, considerándola potencialmente dotada de fuerzas y capacidad para generar soluciones positivas para la vida de niños y jóvenes.

\section{Bibliografía}

ARCHER M.S. (2003), Structure, agency and internal conversation, Cambridge, Cambridge University,

ASHLEY C., HOLTON L., HORAN H. E WIFFIN J. (2006), The family group conference toolkit, London, Family Rights Group.

ASHLEY C. E NIXON P. (2007), Family group conference - Where next?, London, Family Rights Group.

BARNES M. E PRIOR D. (2009), Subversive Citizens. Power agency and resistance in public service, Bristol, The Policy Press.

BURFORD G. (2010), Famiglie che partecipano, Lavoro sociale, vol. 10, n. 1, pp. 7-18.

BURFORD G. (2011), "Il lavoro con le famiglie nella tutela minorile: le Family Group Conference", in P. Donati, Folgheraiter e M.L. Raineri (a cura di), La tutela dei minori. Nuovi scenari relazionali, Trento, Erickson.

CONNOLLY M. (1999), Effective partecipatory practice: Family group conferencing in child protection, NewYork, Aldine de Gruyter.

CONNOLY M. (2006), Fifteen years of Family group conferencing: coordinators talk about their experience in Aotearoa New Zealand, British Journal of Social Work, vol. 36, n. 4, pp. 523-540.

DALRYMPLE J. (2002) Family Group Conferences and youth advocacy: the participation of children and young people in family decision making, European Journal of Social Work, Vol. 5, n. 3, pp. 287-299.

DONATI P. (2009), Teoria relazionale della società: i concetti base, Milano, Franco Angeli.

FOLGHERAITER F. (2004), Relational social work: toward networking and societal practices, London; Jessica Kingsley Publishers.

FOLGHERAITER F. (2006), Il fronteggiamento delle famiglie: per una visione societaria del benessere, Lavoro sociale, vol. 6, n. 1, pp. 7-12.

FOLGHERAITER F. (2011), Fondamenti di metodologia relazionale: la logica sociale dell'aiuto, Trento, Erickson.

MACI F. (2008), Insieme per proteggere Laura: un'esperienza di lavoro di rete nella tutela minorile, Lavoro Sociale, vol. 8, n. 1, pp. 121-130.

MACI F. (2011), Lavorare con le famiglie nella tutela minorile. Il modello delle Family group conference, Trento, Erickson.
MARSH P. E CROW G. (1998), Family group conference in Child Welfare, Oxford, Blackwell Science Ltd.

MERKEL-HOLGUIN L. (2004), Sharing power with the people: Family group conferencing as a democratic experiment, Journal of Sociology and Social Welfare, vol. 1, pp. 155-173.

MORRIS K. (2004), Le famiglie al centro della rete: funziona?, Lavoro Sociale, vol. 4, n. 3, pp. 327-336.

MORRIS K., BURFORD G. (2009), "Family decision making: new space for partecipation and resistance". In Barnes M. e Prior D., Subversive citizens. Power, agency and resistance in publicservices, Bristol, Policy, pp. 129 $-135$.

NIXON P., BURFORD G., QUINN A. E EDELBAUM J. (2005), A survey of international practice, policy \& research on Family group conference and related practices, Englewood, American Humane Association.

PARTON N. E O' BYRNE P. (2000), Constructive social work. Towards a new practice, Basingostoke, Palgrave.

WILLIAMS F. (2004), Rethinking family, London, Caouste Gulbenkian Foundation. 



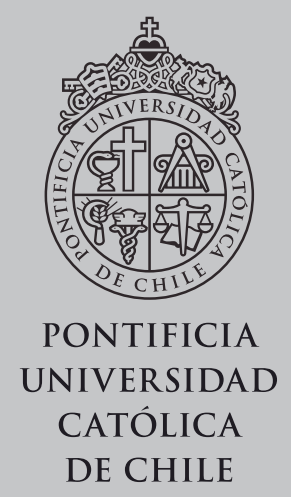

